Collective and intrinsic degrees of freedom in the Heisenberg ferromagnet

This content has been downloaded from IOPscience. Please scroll down to see the full text.

1989 J. Phys. A: Math. Gen. 22703

(http://iopscience.iop.org/0305-4470/22/6/021)

View the table of contents for this issue, or go to the journal homepage for more

Download details:

IP Address: 193.136.215.65

This content was downloaded on 17/04/2017 at 17:00

Please note that terms and conditions apply.

You may also be interested in:

Quantum phase transitions in the collective degrees of freedom: nuclei and other many-body systems Pavel Cejnar and Pavel Stránský

The renormalisation group approach to scaling in physics

D J Wallace and R K P Zia

Microscopic theory of the nuclear collective model

D J Rowe

The many-nucleon theory of nuclear collective structure and its macroscopic limits: an algebraic perspective

D J Rowe, A E McCoy and M A Caprio

A complete Bose transformation in magnetic systems

Ruibao Tao

Exact Bose expansion for general spin

I Goldhirsch

Selfconsistently renormalized spin-wave approximation for some two-dimensional magnetic systems

E Rastelli, A Tassi and L Reatto

Theory of first-order phase transitions

K Binder

Quantum frustration in organic Mott insulators

B J Powell and Ross H McKenzie 


\title{
Collective and intrinsic degrees of freedom in the Heisenberg ferromagnet
}

\author{
J da Providência, C Fiolhais and M Brajczewska \\ Departamento de Fisica, Universidade de Coimbra, P-3000 Coimbra, Portugal
}

Received 25 April 1988, in final form 21 September 1988

\begin{abstract}
A separation between collective and intrinsic degrees of freedom in the Heisenberg model of a ferromagnet at a finite temperature is achieved by reformulating the thermal boson expansion previously obtained by the present authors. In the new approach, all the collective modes (spin waves) have wavenumbers lower than a certain value. The intrinsic energy turns out to be the usual mean-field energy minus a correlation energy due to the excitations of spin waves. The influence of the cutoff in momentum space on the critical temperature is studied and the temperature dependence of the spin-wave renormalisation factor and the magnetisation are calculated.
\end{abstract}

\section{Introduction}

Boson expansions are powerful techniques which aim at studying quantal fluctuations of physical systems around a state of stable equilibrium described in the framework of the mean-field approximation.

In the case of magnetically ordered solids, the method consists basically of replacing the system of interacting spins by a system of bosons, which for all purposes is equivalent, if no approximations are introduced. Fourier transforms of spin operators are mapped into boson operators

$$
A \rightarrow(A)_{\mathrm{B}}
$$

in such a way that if

$$
[A, B]=C
$$

then

$$
\left[(A)_{\mathrm{B}},(B)_{\mathrm{B}}\right]=(C)_{\mathrm{B}} .
$$

We also require that Hermiticity be preserved, i.e.

$$
\left(A^{+}\right)_{\mathrm{B}}=(A)_{\mathrm{B}}^{\dagger} \text {. }
$$

To these conditions we add the requirement that the expectation values of the spin observables in the mean-field ground state $|0\rangle$ be equal to the expectation values of the corresponding boson images in the boson vacuum $\mid 0)$ :

$$
\langle 0|A| 0\rangle=\left(0\left|(A)_{\mathrm{B}}\right| 0\right) \text {. }
$$

By mean-field ground state we mean the independent-particle state (antisymmetrised product of single-spin states) which minimises the energy of the system. In the case of a ferromagnetic system, each single-spin state which enters the ground state has the lowest possible value for $S^{z}$. 
Clearly, boson expansion techniques lead to the replacement of the Hilbert space of the physical system under study by an appropriate subspace-the so-called physical subspace-of the entire boson Hilbert space. The operators representing spin observables are replaced by boson operators. The physics of the problem remains unchanged when going to the boson representation. The Holstein-Primakoff expansion [1] is the classical example of a boson expansion of spin systems, although some other expansions have also been proposed (Dyson-Maleev [23], Schwinger [4], etc).

The method of thermal boson expansion is intended to extend the standard boson expansions (where the ground state $|0\rangle$ is a pure state) to the case of mixed states. This aim is achieved by replacing the expectation value with respect to the mean-field ground state $|0\rangle$ by the statistical average with respect to an independent-particle density matrix $\mathscr{D}_{0}$, such that

$$
\operatorname{Tr}\left(\mathscr{D}_{0} A\right)=\left(0\left|(A)_{\mathrm{B}}\right| 0\right)
$$

for all relevant observables $A$.

The philosophy of the present approach relies heavily on the idea of the separation of collective from intrinsic degrees of freedom, the collective degrees of freedom being bosonised and the intrinsic degrees of freedom being treated in the mean-field approximation.

By bosonisation of the spin degrees of freedom we mean a bosonic realisation of the specific representation of the Lie algebra of the spin operators. It should be kept in mind that the above-mentioned representation is temperature dependent.

At zero temperature, we are dealing with an irreducible representation of the Lie algebra (1.2), but the same does not happen at finite temperature (i.e. for a mixed state). Therefore, the bosonic realisation of the Lie algebra (1.3) is no longer an irreducible representation [5].

In [6] we have applied the method of thermal boson expansion to the Heisenberg ferromagnet with $S=\frac{1}{2}$, having obtained a value of the critical temperature in good agreement with the most accurate value known and even better than the results obtained within the conventional approaches. This temperature has appeared as the maximal attainable solution of a self-consistent equation. Although the phase transition is not continuous (due to the truncation of the boson expansion) the result that a theory of 'free' spin waves could be extended up to the vicinity of the critical point was encouraging.

In [7] we have used the same method to describe systems with spins $S \geqslant 1$. The agreement with experimental data requires in this case a convenient cutoff in momentum space. This reduction of the phase space available for the dynamics is usually invoked in boson expansions, since their rate of convergence is improved when only excitations with large wavelengths are considered.

In the present work we implement a consistent separation between intrinsic and collective degrees of freedom in the Heisenberg model, trying to elucidate the necessity for the momentum cutoff and its influence on some physical quantities. We restrict our considerations to the case $S=\frac{1}{2}$.

We therefore split the Heisenberg Hamiltonian into intrinsic and collective terms. This separation is carried out along the lines of works initiated in the late $19.50 \mathrm{~s}$ in the framework of models of collective behaviour of plasmas and atomic nuclei. It is known that the phenomena of collective quantal motion which are found in nuclear as well as in solid state physics, may be described by unified many-body techniques. 
In $\S 2$ we present a brief review of the method for $S=\frac{1}{2}$ (we refer the reader to [7] for the case $S \geqslant 1$ ).

In $\S 3$ we perform the separation of the Hamiltonian into an intrinsic and a collective part. We observe that this improvement is not a minor detail since it plays a relevant role in overcoming conceptual inconsistencies, such as overestimating the contributions of some degrees of freedom. We show that the main difference with respect to our previous result lies in the subtraction of an appropriate term from the mean-field energy. In the same section we also discuss the statistical mechanics of the new approach and compare the results with those found in the literature on thermal magnons.

In $\S 4$, we present some numerical results, describing the influence of the cutoff on some physical observables. We present the conclusions in $\S 5$.

\section{Review of the method}

Let us consider the Heisenberg model of a lattice of coupled identical spins. We assume that the common value of all spins is $S=\frac{1}{2}$ and that the interaction is restricted to the nearest neighbours. The generalisation of our results to higher spins is straightforward (see [7]). The Hamiltonian may be written

$$
\mathscr{H}=-J \sum_{j, l}^{\prime} \boldsymbol{S}_{j} \cdot \boldsymbol{S}_{l}
$$

where $J$ is the exchange integral and $S_{j}$ is the spin operator of the electron in the site $j$. The prime on the summation sign reminds us that $j$ runs over all $N$ lattice sites while $l$ runs only over the $z$ nearest neighbours of the site $j$. It is convenient to consider the lattice Fourier transforms of the operators $\boldsymbol{S}_{j}$

$$
\begin{aligned}
S_{k}^{ \pm} & =N^{-1 / 2} \sum_{j} \exp \left( \pm \mathrm{i} \boldsymbol{k} \cdot \boldsymbol{R}_{j}\right) S_{j}^{ \pm} \\
S_{k}^{z} & =N^{-1 / 2} \sum_{j} \exp \left(\mathrm{i} \boldsymbol{k} \cdot \boldsymbol{R}_{j}\right) S_{j}^{z}
\end{aligned}
$$

where $\boldsymbol{R}_{j}$ is the vector from the origin to the site $j$. The operators $S_{k}^{ \pm}=S_{k}^{x} \pm \mathrm{i} S_{k}^{y}$ satisfy the commutation relations

$$
\begin{aligned}
& {\left[S_{k}^{+}, S_{k^{\prime}}^{-}\right]=2 N^{-1 / 2} S_{k-k^{\prime}}^{z}} \\
& {\left[S_{k}^{z}, S_{k^{\prime}}^{ \pm}\right]= \pm N^{-1 / 2} S_{k \pm k^{\prime}}^{ \pm} .}
\end{aligned}
$$

In terms of the operators $S_{k}^{ \pm}$and $S_{k}^{z}$ the Hamiltonian (2.1) may be rewritten as

$$
\mathscr{H}=-J z \sum_{k} \gamma_{k}\left[\frac{1}{2}\left(S_{k}^{+} S_{k}^{-}+S_{k}^{-} S_{k}^{+}\right)+S_{k}^{z} S_{-k}^{z}\right]
$$

where

$$
\gamma_{k}=z^{-1} \sum_{l} \exp \left(\mathrm{i} \boldsymbol{k} \cdot \boldsymbol{\delta}_{l}\right)
$$

Here, $\delta_{l}$ denotes the vector from the site $j$ to the neighbouring site $l$.

We are looking for the mapping of the operators $S_{k}^{ \pm}$and $S_{k}^{z}$ onto functions of operators $B_{k}$ and $B_{k}^{+}$which satisfy boson commutation relations

$$
\left[B_{k}, B_{k^{\prime}}^{\dagger}\right]=\delta_{k, k^{\prime}} \quad\left[B_{k}, B_{k^{\prime}}\right]=\left[B_{k}^{+}, B_{k^{\prime}}^{\dagger}\right]=0 .
$$


We denote by $(0)$ the vacuum of the Fock space in which the operators $B_{k}$ and $B_{k}^{\dagger}$ are defined, so that $\left.B_{k} \mid 0\right)=0$. The mapping should preserve the commutation relations (2.4) and (2.5), so that

$$
\begin{aligned}
& {\left[\left(S_{k}^{+}\right)_{\mathrm{B}},\left(S_{k^{\prime}}^{-}\right)_{\mathrm{B}}\right]=2 N^{-1 / 2}\left(S_{k-k^{\prime}}^{z}\right)_{\mathrm{B}}} \\
& {\left[\left(S_{k}^{z}\right)_{\mathrm{B}},\left(S_{k^{\prime}}^{ \pm}\right)_{\mathrm{B}}\right]= \pm N^{-1 / 2}\left(S_{k \pm k^{\prime}}^{ \pm}\right)_{\mathrm{B}}}
\end{aligned}
$$

as well as the expectation value with respect to a mean-field density matrix $\mathscr{D}_{0}$ describing the 'mixed ground state'

$$
\begin{aligned}
& \operatorname{Tr}\left(\mathscr{D}_{0} S_{k}^{ \pm}\right)=\left(0\left|\left(S_{k}^{ \pm}\right)_{\mathrm{B}}\right| 0\right) \\
& \operatorname{Tr}\left(\mathscr{D}_{0} S_{k}^{z}\right)=\left(0\left|\left(S_{k}^{z}\right)_{\mathrm{B}}\right| 0\right) .
\end{aligned}
$$

By mixed ground state we mean the statistical state represented by a density matrix $\mathscr{D}_{0}$ such that

$$
\operatorname{Tr}\left(\mathscr{D}_{0} \mathscr{H}\right) \leqslant \operatorname{Tr}\left(U \mathscr{D}_{0} U^{\dagger} \mathscr{H}\right)
$$

for all unitary operators $U$ which preserve the independent particle character of $\mathscr{D}_{0}$. Therefore

$$
U=\exp (\mathrm{i} T)
$$

where $T$ is an arbitrary Hermitian one-body operator.

Since $\mathscr{D}_{0}$ describes a mean-field or independent-particle mixed state, it may be written in the form

$$
\mathscr{D}_{0}=A \exp \left(\alpha \sum_{j} S_{j}^{z}\right)=A \exp \left(\alpha N^{1 / 2} S_{0}^{z}\right)
$$

where $S_{0}^{z}=S_{k_{k=0}}^{z}$ and $A$ and $\alpha$ are constants related to each other by the normalisation condition $\operatorname{Tr} \mathscr{D}_{0}=1$. We denote the probability of finding a spin up by $p=A^{1 / N} \exp \left(\frac{1}{2} \alpha\right)$ and the probability of finding a spin down by $q=1-p=A^{1 / N} \exp \left(-\frac{1}{2} \alpha\right)$. The density matrix $\mathscr{D}_{0}$ depends on a single parameter which may be taken to be the difference $X=p-q$

$$
X=\tanh \left(\frac{1}{2} \alpha\right) \text {. }
$$

Straightforward calculations lead to the following results:

$$
\begin{aligned}
& \operatorname{Tr}\left(\mathscr{D}_{0} S_{k}^{ \pm}\right)=0 \\
& \operatorname{Tr}\left(\mathscr{D}_{0} S_{k}^{z}\right)=\frac{1}{2} N^{1 / 2} X \delta_{k, 0} \\
& \operatorname{Tr}\left(\mathscr{D}_{0}\left[S_{k}^{+}, S_{k^{\prime}}^{-}\right]\right)=X \delta_{k, k^{\prime}} \\
& \operatorname{Tr}\left(\mathscr{D}_{0}\left[S_{k}^{+}, S_{k^{\prime}}^{+}\right]\right)=\operatorname{Tr}\left(\mathscr{D}_{0}\left[S_{k}^{z}, S_{k^{\prime}}^{ \pm}\right]\right)=0 \\
& \operatorname{Tr}\left(\mathscr{D}_{0}\left[\left[S_{k}^{z}, S_{k^{\prime}}^{+}\right], S_{k^{\prime \prime}}^{-}\right]\right)=N^{-1 / 2} X \delta_{k+k^{\prime}, k^{\prime \prime}} \\
& \operatorname{Tr}\left(\mathscr{D}_{0}\left[\left[S_{k}^{z}, S_{k^{\prime}}^{+}\right], S_{k^{\prime \prime}}^{+}\right]\right)=0 .
\end{aligned}
$$

We require that the equations $(2.17)-(2.22)$ be preserved by the boson map

$$
\begin{aligned}
& \left(0\left|\left(S_{k}^{ \pm}\right)_{\mathrm{B}}\right| 0\right)=0 \\
& \left(0\left|\left(S_{k}^{z}\right)_{\mathrm{B}}\right| 0\right)=\frac{1}{2} N^{1 / 2} X \delta_{k, 0} \\
& \left(0\left|\left[\left(S_{k}^{+}\right)_{\mathrm{B}},\left(S_{k^{\prime}}^{-}\right)_{\mathrm{B}}\right]\right| 0\right)=X \delta_{k, \boldsymbol{k}^{\prime}} \\
& \left(0\left|\left[\left(S_{k}^{+}\right)_{\mathrm{B}},\left(S_{k^{\prime}}^{+}\right)_{\mathrm{B}}\right]\right| 0\right)=\left(0\left|\left[\left(S_{k}^{z}\right)_{\mathrm{B}},\left(S_{k^{\prime}}^{ \pm}\right)_{\mathrm{B}}\right]\right| 0\right)=0 \\
& \left(0\left|\left[\left[\left(S_{k}^{z}\right)_{\mathrm{B}},\left(S_{k^{\prime}}^{+}\right)_{\mathrm{B}}\right],\left(S_{k^{\prime \prime}}^{-}\right)_{\mathrm{B}}\right]\right| 0\right)=N^{-1 / 2} X \delta_{k+\boldsymbol{k}^{\prime}, \boldsymbol{k}^{\prime \prime}} \\
& \left(0\left|\left[\left[\left(S_{k}^{z}\right)_{\mathrm{B}},\left(S_{k^{\prime}}^{+}\right)_{\mathrm{B}}\right],\left(S_{\boldsymbol{k}^{\prime \prime}}^{+}\right)_{\mathrm{B}}\right]\right| 0\right)=0 .
\end{aligned}
$$


These requirements establish the boson expansions

$$
\begin{aligned}
& \left(S_{k}^{+}\right)_{\mathrm{B}}=X^{1 / 2} B_{k}+\ldots \quad\left(S_{k}^{-}\right)_{\mathrm{B}}=X^{1 / 2} B_{k}^{\dagger}+\ldots \\
& N^{1 / 2}\left(S_{k}^{z}\right)_{\mathrm{B}}=\frac{1}{2} N X \delta_{k, 0}-\sum_{k^{\prime}} B_{k+k^{\prime}}^{\dagger} B_{k^{\prime}}+\ldots
\end{aligned}
$$

Analogously, the conditions

$$
\begin{aligned}
& \left.\operatorname{Tr}\left(\mathscr{D}_{0} \mathscr{H}\right)=(0 \mid \mathscr{H})_{\mathrm{B}} \mid 0\right)=-\frac{1}{4} J N X^{2} z \\
& \operatorname{Tr}\left(\mathscr{D}_{0}\left[\mathscr{H}, S_{k}^{ \pm}\right]\right)=\left(0\left|\left[(\mathscr{H})_{\mathrm{B}},\left(S_{k}^{ \pm}\right)_{\mathrm{B}}\right]\right| 0\right)=0 \\
& \operatorname{Tr}\left(\mathscr{D}_{0}\left[\left[\mathscr{H}, S_{k}^{+}\right], S_{k^{\prime}}^{-}\right]\right)=\left(0\left|\left[\left[(\mathscr{H})_{\mathrm{B}},\left(S_{k}^{+}\right)_{\mathrm{B}}\right],\left(S_{k^{\prime}}^{-}\right)_{\mathrm{B}}\right]\right| 0\right)=-J z\left(1-\gamma_{k}\right) X^{2} \delta_{k, \boldsymbol{k}^{\prime}} \\
& \operatorname{Tr}\left(\mathscr{D}_{0}\left[\left[\mathscr{H}, S_{k}^{+}\right], S_{k^{\prime}}^{+}\right]\right)=\left(0\left|\left[\left[(\mathscr{H})_{\mathrm{B}},\left(S_{k}^{+}\right)_{\mathrm{B}}\right],\left(S_{k^{\prime}}^{+}\right)_{\mathrm{B}}\right]\right| 0\right)=0
\end{aligned}
$$

etc, lead to the boson expansion of the Hamiltonian

$$
(\mathscr{H})_{\mathrm{B}}=-\frac{1}{4} N J z X^{2}+\sum_{k} J z\left(1-\gamma_{k}\right) X B_{k}^{\dagger} B_{k}+\ldots
$$

This expansion, when taken to all orders, is a bosonic realisation of the original Hamiltonian which is able to reproduce the same results. It is important to estimate the rate of convergence of the boson expansion. This may be done by evaluating quantities such as

$$
\begin{aligned}
\operatorname{Tr}\left(\mathscr{D}_{0}\left[S_{\boldsymbol{k}^{\prime \prime \prime}}^{-},\left[S_{\boldsymbol{k}^{\prime \prime}}^{-}, S_{\boldsymbol{k}^{\prime}}^{+} S_{\boldsymbol{k}}^{+}\right]\right]\right) & =X^{2}\left(\delta_{k^{\prime}, \boldsymbol{k}^{\prime \prime}} \delta_{k, \boldsymbol{k}^{\prime \prime}}+\delta_{k, \boldsymbol{k}^{\prime}} \delta_{\boldsymbol{k}^{\prime \prime}, \boldsymbol{k}^{\prime \prime \prime}}\right)+\mathscr{O}(1 / N) \\
& =\left(0 \mid\left[B_{\boldsymbol{k}^{\prime \prime}}^{+},\left[B_{k^{\prime \prime}}^{\dagger}, B_{k^{\prime}} B_{k}\right]\right] 0\right)+\mathscr{O}(1 / N)
\end{aligned}
$$

This kind of result clearly indicates that the replacement of the operators $S_{k}^{ \pm}$by boson operators is a good approximation to order $1 / N$. Therefore, our method may break down only when it is applied to the description of highly excited states, for which the number of bosons is of order $N$.

\section{Intrinsic and collective degrees of freedom}

In this section we are interested in decomposing the Hamiltonian into intrinsic and collective parts, starting from the bosonised Heisenberg Hamiltonian obtained in the last section.

In contrast with the treatment given in [6], we would like to consider now a kind of boson expansion where only some degrees of freedom are bosonised (collective degrees of freedom). All collective bosons have wavevectors lower than a given value $k_{\mathrm{m}}<k_{\mathrm{Bz}}$, with $k_{\mathrm{Bz}}$ being the Brillouin boundary radius. The remaining degrees of freedom are called intrinsic. We are going to use the mean-field approximation to account for the intrinsic dynamics and to look at the influence of the momentum cutoff on the critical point (maximal attainable temperature) and some physical observables (spin-wave renormalisation factor, magnetisation).

The intrinsic energy calculated in the mean-field approach will turn out to be simply the mean-field energy of the total Hamiltonian minus the energy of the magnons, calculated in the spin space.

In order to demonstrate this statement, we take advantage of a procedure for separating the Hamiltonian into collective and intrinsic parts which has been proposed by Tomonaga [8], who was interested in a unified approach to different examples of 
collective motion (nuclear surface vibrations and charge oscillations of the electron gas). The method has since been used to handle similar problems (e.g. the Villars formalism for describing nuclear rotations [9]).

In Tomonaga's approach, the Hamiltonian which one wants to study is expanded in such a way that only some degrees of freedom are bosonised, while the remaining are re-expressed as many-particle degrees of freedom. Up to the harmonic order in the collective bosons the bosonised Heisenberg Hamiltonian is written as

$$
(\mathscr{H})_{\mathrm{B}}=\mathscr{H}^{(0)}+\mathscr{H}^{(1)}+\mathscr{H}^{(2)}+\ldots
$$

with

$$
\begin{aligned}
& \mathscr{H}^{(0)}=\mathscr{H}_{\mathrm{int}} \\
& \mathscr{H}^{(1)}=\sum_{k}^{\prime}\left(G_{k}^{-} B_{k}^{+}+G_{k}^{+} B_{k}\right)
\end{aligned}
$$

and

$$
\mathscr{H}^{(2)}=\sum_{k, k^{\prime}}^{\prime}\left(G_{k k^{\prime}}^{--} B_{k}^{\dagger} B_{k^{\prime}}^{\dagger}+G_{k k^{\prime}}^{++} B_{k} B_{k^{\prime}}+G_{k k^{\prime}}^{-+} B_{k}^{\dagger} B_{k^{\prime}}\right)
$$

where the prime on the summations indicates an imposed cutoff on the wavevectors $\left(|\boldsymbol{k}|<k_{\mathrm{m}}\right)$. The operators $\mathscr{H}_{\mathrm{int}}, G_{k}^{-}, G_{k}^{+}, G_{k k^{\prime}}^{++}, G_{k k^{\prime}}^{--}, G_{k k^{\prime}}^{-+}$are called intrinsic. They are functions of the intrinsic degrees of freedom (intrinsic boson operators) and commute with the collective boson operators, e.g.

$$
\begin{array}{ll}
{\left[\mathscr{H}_{\mathrm{int}}, B_{\boldsymbol{k}}^{\dagger}\right]=\left[\mathscr{H}_{\mathrm{int}}, B_{\boldsymbol{k}}\right]=0} & |\boldsymbol{k}|<k_{\mathrm{m}} \\
{\left[G_{\boldsymbol{k}}^{+}, B_{\boldsymbol{k}^{\prime}}^{\dagger}\right]=\left[G_{k}^{+}, B_{\boldsymbol{k}^{\prime}}\right]=0} & |\boldsymbol{k}|<k_{\mathrm{m}}
\end{array}
$$

with analogous relations for the remaining operators. We remark that (3.1) is nothing more than a way of rewriting (2.35).

Simple algebra shows that $\left(G_{k k^{\prime}}^{--}=G_{k k^{\prime}}^{++}=0\right)$

$$
\begin{aligned}
{\left[(\mathscr{H})_{\mathrm{B}}, B_{k}^{\dagger}\right] } & =G_{k^{\prime}}^{+}\left[B_{k^{\prime}}, B_{k}^{\dagger}\right]+G_{k^{\prime} k^{\prime \prime}}^{++}\left[B_{k^{\prime}}^{\dagger} B_{k^{\prime \prime}}, B_{k}^{\dagger}\right]+\ldots \\
& =G_{k}^{+}+G_{k^{\prime} k}^{-+} B_{k^{\prime}}^{\dagger}+\ldots
\end{aligned}
$$

while

$$
\left[(\mathscr{H})_{\mathrm{B}}, B_{k}\right]=-G_{k}^{-}-G_{k k^{\prime}}^{-+} B_{k^{\prime}}+\ldots
$$

We have then

$$
\begin{aligned}
& G_{k}^{+}=\left[(\mathscr{H})_{\mathrm{B}}, B_{k}^{\dagger}\right]-G_{k^{\prime} k}^{-+} B_{k^{\prime}}^{\dagger}+\ldots \\
& G_{k}^{-}=\left[B_{k},(\mathscr{H})_{\mathrm{B}}\right]-G_{k k^{\prime}}^{-+} B_{k^{\prime}}+\ldots
\end{aligned}
$$

The following double commutators are easily evaluated

$$
\begin{aligned}
& {\left[B_{k^{\prime}},\left[(\mathscr{H})_{\mathrm{B}}, B_{k}^{\dagger}\right]\right]=G_{\boldsymbol{k}^{\prime} \boldsymbol{k}}^{-+}} \\
& {\left[B_{\boldsymbol{k}^{\prime}}^{+},\left[(\mathscr{H})_{\mathrm{B}}, B_{k}\right]\right]=G_{k k^{\prime}}^{-+} .}
\end{aligned}
$$

We now replace the operators $G_{\boldsymbol{k n}^{\prime}}^{-+}$and $G_{\boldsymbol{k}^{\prime} \boldsymbol{k}}^{-+}$by their expectation values with respect to the density matrix $\mathscr{D}_{0}$ (the fluctuations around mean-field values are hopefully negligible) and use the mapping between boson and spin operators $S_{k}^{+} \simeq X^{1 / 2} B_{k}$, and $S_{k}^{-} \simeq X^{1 / 2} B_{k}^{\dagger}$ (these relations are valid to first order):

$$
\begin{aligned}
G_{k k^{\prime}}^{-+} & \simeq(1 / X) \operatorname{Tr}\left(\mathscr{D}_{0}\left[S_{k}^{+},\left[\mathscr{H}, S_{k^{\prime}}^{-}\right]\right]\right) \\
& =(1 / X) \operatorname{Tr}\left(\mathscr{D}_{0}\left[S_{k^{\prime}}^{-},\left[\mathscr{H}, S_{k}^{+}\right]\right]\right) \\
& =J X z\left(1-\gamma_{k}\right) \delta_{k, k^{\prime}}=\omega_{k}(X) \delta_{k, k^{\prime}}
\end{aligned}
$$


We have therefore

$$
\begin{aligned}
& G_{k}^{+}=\left[(\mathscr{H})_{\mathrm{B}}, B_{k}^{+}\right]-\omega_{k}(X) B_{k}^{\dagger}+\ldots \\
& G_{k}^{-}=\left[B_{k},(\mathscr{H})_{\mathrm{B}}\right]-\omega_{k}(X) B_{k}+\ldots
\end{aligned}
$$

Taking again the expectation value after having replaced boson operators by spin operators we conclude that these intrinsic operators are very small. One obtains

$$
\mathscr{H}_{\mathrm{int}} \simeq \mathscr{H}-\sum_{k}^{\prime} \frac{\omega_{k}(X)}{X} S_{k}^{-} S_{k}^{+} .
$$

The smallness of the intrinsic terms $G_{k}^{+}$and $G_{k}^{-}$may be explained by the fact that they describe the coupling between collective and intrinsic variables, which is small. In our case these operators are of at least second order in the intrinsic boson operators, due to momentum conservation.

The final result (3.16) says that a collective energy term (energy corresponding to the collective variables) must be subtracted from the total energy calculated in the mean-field approximation. We are thus sure that the collective degrees of freedom are not being counted twice in the energy.

\section{Results}

We may now evaluate the free energy of the system and, with the aid of the Peierls variational principle, derive an equation which expresses the parameter determining the statistical mixture in terms of the temperature. We follow the approach of [6], extended by the addition of the new term obtained in the previous section and restricting all summations in $\boldsymbol{k}$ space.

The free energy may be written

$$
F=F_{0}+F_{2}
$$

where $F_{0}$ is the intrinsic contribution in the mean-field approximation, now corrected with respect to expression (3.2) of [6]:

$$
\begin{gathered}
F_{0}=E_{0}-T S_{0}-\Delta E_{0}=-\frac{1}{4} N J X^{2} z+\frac{1}{2} N k_{\mathrm{B}} T\left\{(1+X) \log \left[\frac{1}{2}(1+X)\right]\right. \\
\left.-(1-X) \log \left[\frac{1}{2}(1-X)\right]\right\}-\Delta E_{0}
\end{gathered}
$$

with

$$
\Delta E_{0}=\sum_{k}^{\prime} \frac{\omega_{k}(X)}{X} \operatorname{Tr}\left(\mathscr{D}_{0} S_{k}^{-} S_{k}^{+}\right)
$$

and the collective contribution in the independent-boson approximation is

$$
\begin{aligned}
F_{2}=E_{2}-T S_{2} & =\sum_{k}^{\prime} \omega_{k}(X) n_{k}(X)+k_{\mathrm{B}} T \sum_{k}^{\prime}\left[n_{k}(X) \log n_{k}(X)\right. \\
& \left.-\left(1+n_{k}(X)\right) \log \left(1+n_{k}(X)\right)\right]
\end{aligned}
$$

where

$$
n_{k}(X)=1 /\left[\exp \left(\beta \omega_{k}(X)\right)-1\right]
$$

is the number of magnons and $\beta=1 / k_{\mathrm{B}} T$, with $k_{\mathrm{B}}$ being the Boltzmann constant. 
The parameter $X$ is determined by imposing the condition of extremum for the free energy

$$
\mathrm{d} F / \mathrm{d} X=0
$$

yielding for a simple cubic lattice $(z=6)$

$$
X=\tanh \left[\frac{3 J}{k_{\mathrm{B}} T}\left(X-\frac{1}{3 N J} \sum_{k}^{\prime} \omega_{k}(1) n_{k}(X)-\frac{1}{6 N J} \sum_{k}^{\prime} \omega_{k}(1)\right)\right]
$$

We remark again that the modifications relative to [6] are the inclusion of a new term and the restriction in the summations.

The magnetisation is given by the expectation value of

$$
(M)_{\mathrm{B}}=\frac{g \mu_{\mathrm{B}} f}{N a^{3}}\left(\sum_{j} S_{j}^{z}\right)_{\mathrm{B}}=\frac{g \mu_{\mathrm{B}} f}{N a^{3}}\left(\frac{N}{2} X-\sum_{k}^{\prime} B_{k}^{\dagger} B_{k}\right)
$$

where $g$ is the Lande factor, $\mu_{\mathrm{B}}$ is the Bohr magneton, $a$ is the lattice constant and $f=1,2$ and 4 for SC, BCC and FCC lattices respectively.

The reduced magnetisation is

$$
\frac{M(X)}{M(1)}=X-\frac{2}{N} \sum_{k}^{\prime} n_{k}(X)
$$

Equation (4.7) has been solved for $X$ and the magnetisation has been obtained by inserting $X$ into (4.9). The summations in momentum space have been done numerically by evaluating an integral whose upper limit corresponds to the cutoff value.

The transcendental equation (4.7) only has solutions for temperatures lower than a maximum value, which we identify with the critical temperature $T_{c}$. The curve for $X$ re-enters near $T_{c}$, i.e. in that region two solutions $X$ are found for the same temperature. The magnetisation is also double valued near $T=T_{\mathrm{c}}$, the upper branch corresponding to a lower free energy. This type of behaviour (coexistence of two phases) is characteristic of first-order phase transitions [10]. It is interesting to note that a first-order phase transition for the Heisenberg model has been reported in a mean-field approach to the phenomenon of magnetostriction, which brings about a renormalisation of the exchange constant in terms of the magnetisation [11].

In figure 1 the dependence of the critical temperature on the value of the cutoff is shown. We note that although the curves shown agree for small values of the cutoff, as they should, since switching off the magnons effectively reproduces the Weiss mean-field theory, the two curves are clearly different when the cutoff approaches the Brillouin edge. In fact, in the present approach it is not even possible to consider the wavevector corresponding to the Brillouin zone limit (there is no solution above $0.9 k_{\mathrm{Bz}}$ ). The conclusion, therefore, is that a cutoff momentum is indeed meaningful.

In figure 1 the lower horizontal line indicates the 'exact' critical temperature (by 'exact' we mean the value obtained by a conventional high-temperature expansion for the magnetic susceptibility, incorporating a high number of terms [12]) and the upper line indicates the approximation to the critical temperature calculated by Rushbrooke and Wood (the Rushbrooke-Wood empirical formula yields very good results for high spins, as discussed in [7]).

In contrast to the previous formulation, in which good prediction of the critical temperature was found at $k_{\mathrm{m}}=k_{\mathrm{Bz}}$, the 'exact' critical point is now achieved for a cutoff value of $0.61 k_{\mathrm{Bz}}$. That means that almost half of the phase space should be excluded. Although the cut-off $k_{\mathrm{m}}$ has been adjusted to known results, we point out 


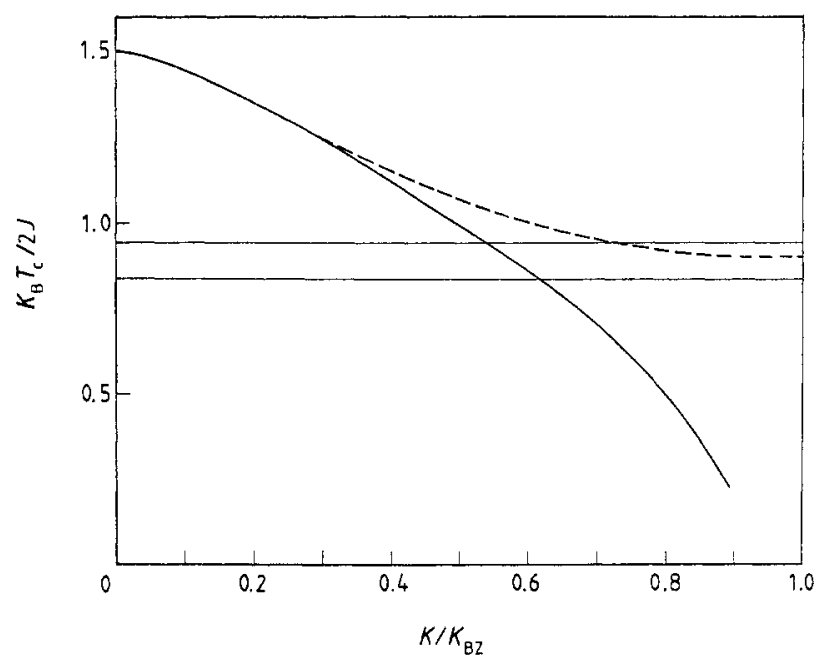

Figure 1. Reduced critical temperature as a function of the reduced cutoff momentum (in units of the Brillouin momentum) for a simple cubic lattice and spin $\frac{1}{2}$. The full curve represents calculations based on the corrected thermal boson expansion-there is no solution above $k / k_{\mathrm{Bz}}=0.9$. The broken curve is the result obtained without subtracting the correction to the mean-field energy (see [6]). The upper horizontal line indicates the critical temperature as given by the Rushbrooke-Wood formula, while the lower corresponds to the exact critical temperature.

that it is possible to indicate some bounds for $k_{\mathrm{m}}: 0.4 k_{\mathrm{Bz}} \leqslant k_{\mathrm{m}} \leqslant 0.68 k_{\mathrm{Bz}}$. For $k_{\mathrm{m}}<$ $0.4 k_{\mathrm{Bz}}$, the magnetisation is no longer re-entrant, having a negative slope at $T=T_{\mathrm{c}}$ (when $M=0$ ), while for $k_{\mathrm{m}}>0.68 k_{\mathrm{Bz}}$, the lower branch of the magnetisation does not reach zero.

The results for the renormalisation factor $X$ and the reduced magnetisation are shown in figures 2 and 3, respectively (the cutoff is fixed, once and for all, as being $k_{\mathrm{m}}=0.61 k_{\mathrm{Bz}}$ ), in comparison with the results of [6]. The discrepancy between both results is only significant near the critical point. This fact is easy to understand since the thermodynamics is dominated by magnons near absolute zero and by both the mean-field and magnons at high temperatures, so any correction to the mean-field result will have a clear influence on the physical observables. For instance, at $T=T_{\mathrm{c}}$ both $X$ and $M(X) / M(1)$ have a lower value than in [6].

Table 1 displays information on the values of the different terms which add up to yield the total free energy. We note that the different terms of the expansion for the energy converge rapidly (i.e. $E_{2} \ll\left|E_{0}\right|$ throughout the full range of temperature). Furthermore, we have verified that the first anharmonic term is indeed small when compared with $E_{2}$ and with $\left|E_{0}\right|$. The correction to the mean-field energy is always smaller than $E_{2}$. The ratio $\Delta E_{0} / E_{2}$ does not go beyond $50 \%$. The inequality $E_{2} \neq \Delta E_{0}$ can be explained by the fact that the latter has been evaluated by a statistical average of $S_{k}^{+} S_{k}^{-}$in spin space while the former has been calculated by taking the expectation value of $B_{k}^{+} B_{k}$ in boson space.

At low temperatures the internal energy $U=E_{0}+E_{2}-\Delta E_{0}$ displays the correct asymptotic behaviour $U \sim T^{5 / 2}$, while very close to the critical point it increases abruptly, leading to a singularity in the specific heat.

We would like to comment on the number of magnons. The last column of table 1 shows that this number is always much smaller than the number of spins, as required 


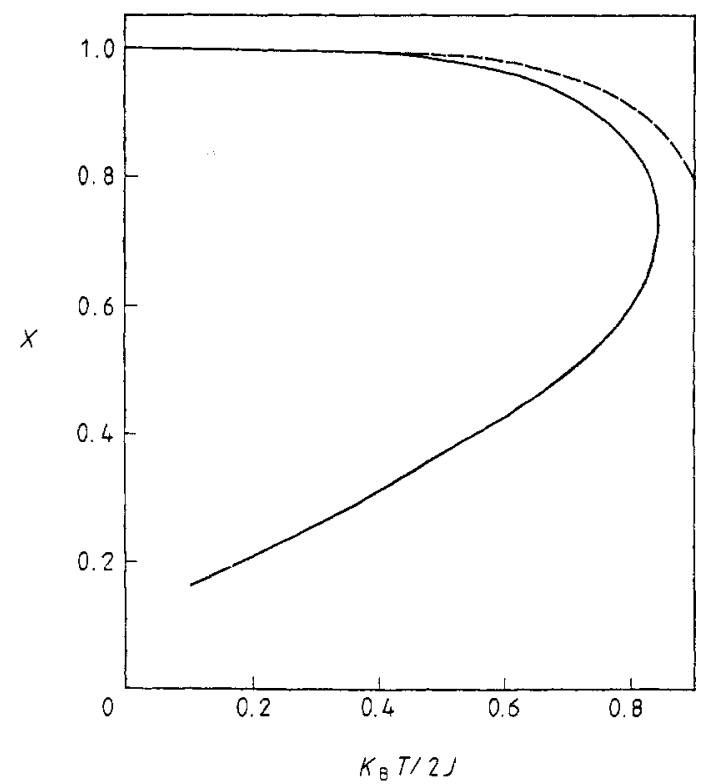

Figure 2. Renormalisation factor $X$ for the spin-wave energy as a function of reduced temperature for a simple cubic lattice and spin $\frac{1}{2}$. The full curve represents calculations based on the corrected thermal boson expansion with the cutoff fixed at $0.61 k_{\mathrm{Bz}}$. The broken curve is the result published in [6] (the lower branch is not shown).

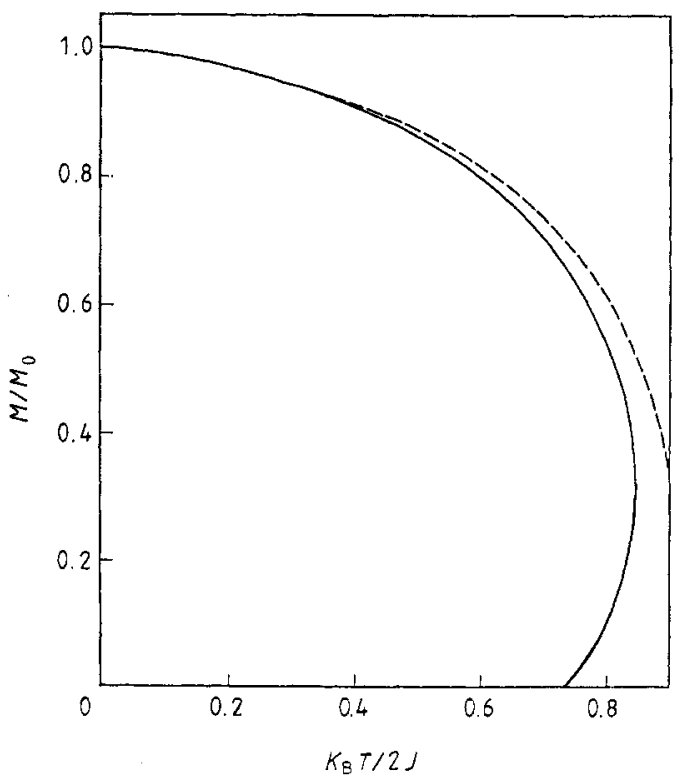

Figure 3. Reduced magnetisation $M / M_{0}$ as a function of the reduced temperature for a simple cubic lattice and spin $\frac{1}{2}$. The full curve represents calculations based on the corrected thermal boson expansion with the cutoff momentum fixed at $0.61 k_{\mathrm{Bz}}$. The broken curve is the result published in [6] (the lower branch is not shown). 
Table 1. Values (in units of $2 J N \times 10^{-3}$ ) of all the contributions to the free energy and the ratio between the number of magnons and the total number of particles for different temperatures. The momentum cutoff is fixed at $k / k_{\mathrm{Bz}}=0.61$.

\begin{tabular}{lccrrrl}
\hline$T / T_{\mathrm{c}}$ & $E_{0}$ & $T S_{0}$ & $E_{2}$ & $T S_{2}$ & $\Delta E_{0}$ & $\Sigma_{k}^{\prime} n_{k} / N$ \\
\hline 0.2 & -748 & $5 \times 10^{-4}$ & 1.6 & 2.6 & $1 \times 10^{-5}$ & 0.01 \\
0.4 & -747 & 1.2 & 9.3 & 15.2 & 0.2 & 0.03 \\
0.6 & -735 & 18.6 & 25.3 & 42.1 & 2.1 & 0.06 \\
0.8 & -666 & 87.6 & 50.8 & 87.5 & 10.1 & 0.11 \\
1.0 & -397 & 331.9 & 97.1 & 181.7 & 47.2 & 0.20 \\
\hline
\end{tabular}

to assure the convergence of the boson expansion. The fact that the number of magnons is always lower than $N$ represents an advantage over the random phase approximation (RPA) results as discussed below.

Essentially two methods have been proposed for extending spin waves to finite temperatures. In the Bloch approach [13], the spin waves are renormalised by the energy (dynamical renormalisation), this procedure leading to a phase transition similar to ours. On the other hand, the RPA method $[14,15]$, which departs from a somewhat ad hoc decoupling of the temperature-dependent spin Green function, consists of a renormalisation in terms of the mean field (kinematical renormalisation). An important drawback of the latter procedure is that the renormalisation factor vanishes at the transition point, there being then an infinite number of magnons $[16,17]$.

Our thermal boson method combines mean-field and boson contributions to the free energy. As in the above-mentioned approaches, the results near the transition point are not close enough to experimental values. We obtain a first-order phase transition instead of a second-order one. The magnetisation does not go to zero at $T=T_{\mathrm{c}}$, so that we cannot define critical exponents (the concept of universality is only pertinent to continuous phase transitions). The descriptions near the critical temperature provided by the available theories of finite-temperature magnons should be regarded as more qualitative than quantitative. In all these approaches, some continuation of low-temperature results is obtained at intermediate temperatures, the treatment breaking down at a point near the exact critical temperature. We believe that our description of temperature-dependent magnons takes proper account of the kinematical problem (ignored in the energy renormalisation method), being assured that the number of magnons is always lower than $N$ (in contrast to the RPA method). We have obtained $(1 / N) \Sigma_{k}^{\prime} n_{k}=0.20$ at $T=T_{\mathrm{c}}$.

\section{Conclusions}

In this paper we have developed the theory of free spin waves at finite temperatures introduced in [6]. We refer to the spin waves as 'free' since no explicit interaction between them through anharmonic terms is considered. Notwithstanding, they do effectively interact with each other. This interaction is described by a spin-wave renormalisation factor which is determined by a variational principle for the total free energy. According to the usual terminology, this interaction has a 'kinematical' origin [2]. One conclusion of our work is that the kinematical interaction is very important near the phase transition.

Our technique is called a 'thermal boson expansion' since there is a mapping of spin observables onto boson operators which is temperature dependent. The expansion 
may be extended by incorporating any desired number of terms. The fact that the operators which are chosen to be mapped are collective (i.e. lattice Fourier transforms of localised spin operators with large wavelengths) is very important, since it assures a good convergence of the expansion and therefore allows for reliable truncations. We have verified that anharmonic terms are not relevant if the number of magnons is small enough. At the same time, the restriction to the collective subspace avoids a large spurious coupling with boson states having no physical counterpart (the boson Hilbert space is infinite in contrast with the spin space) and therefore takes kinematical correlations into account.

We are therefore in agreement with Stoller [18] when he writes that 'any approach to the kinematical problem that does not perform some state space reduction has, $a b$ initio, missed a large part of the physics' or, later and more explicitly, that 'state space reduction is a large part of the kinematical effect'.

We have particularly addressed the problem of avoiding the duplication, in the mean-field dynamics, of the degrees of freedom which are considered in the form of spin waves. This problem, well known in many domains of many-body physics, has so far, to the best of our knowledge, not been studied for the Heisenberg ferromagnet.

Our goal has been accomplished by means of Tomonaga's formalism of separation of the Hamiltonian into intrinsic and collective parts, resulting in some correlation energy associated with the collective motion being subtracted from the total mean-field energy.

The correction to the mean-field energy, although unimportant at low temperatures, increases for high temperatures, in agreement with the fact that the number of magnons increases with the temperature. Therefore it is mainly near the critical point that the picture developed in [6] has been modified. The description of the transition region remains, however, qualitative.

We have analysed the dependence of the critical temperature on the momentum cutoff, concluding that the improved formalism indeed requires such a cutoff. The value of the cutoff remains the only free parameter of the theory, having been adjusted to the known critical temperature. Although the introduction of a cutoff in momentum space has a physical justification, we remark that there seems to be no unambigous way of fixing the cutoff without invoking some extra information.

We have also analysed the case $S \geqslant 1$, which proceeds along similar lines. The conclusion was that no essential modification to the numerical results presented in [7] arose. We stress the fact that for $S \geqslant 1$ there are, besides $S_{k}^{+}$, other possible mechanisms of producing collective excitations, namely mechanisms related to the use of lattice Fourier transforms of some power of the raising operators, e.g. $\left(S_{k}^{+}\right)^{2}$.

\section{Acknowledgment}

This work has been supported by the Instituto Nacional de Investigação Científica, Lisboa, Portugal.

\section{References}

[1] Holstein T and Primakoff H 1940 Phys. Rev. 581698

[2] Dyson F J 1956 Phys. Rev. 102 1217, 1230 
[3] Maleev S V 1958 Sov. Phys.-JETP 6776

[4] Schwinger J 1965 Quantum Theory of Angular Momentum ed L Biedenharn and H Van Dam (New York: Academic) p 229

[5] Okubo S 1974 Phys. Rev. C 102048

Bonatsos D, Klein A and Zhang Q Y 1986 Phys. Lett. 175B 249

[6] Brajczewska M, Fiolhais C and da Providência J 1986 Physica 137A 196

[7] Fiolhais C, Brajczewska M and da Providência J 1987 Physica 147B 249

[8] Tomonaga S 1955 Prog. Theor. Phys. 13467

[9] Villars F 1965 Nucl. Phys. 74353

[10] Binder K 1987 Rep. Prog. Phys. 50783

[11] Stenschke H 1971 Critical Phenomena in Alloys, Magnets and Superconductors ed Mills, Acher and Jaffee (New York: McGraw-Hill) p 125

[12] Rushbrooke G S, Baker G A Jr and Wood F A 1974 Phase Transitions and Critical Phenomena vol 3 , ed C Domb and M S Green (New York: Academic) p 245

[13] Bloch M 1963 J. Appl. Phys. 341151

[14] Bonch-Bruevich V L 1962 The Green Function Method in Statistical Mechanics (Amsterdam: North. Holland)

[15] Tahir-Kheli R A 1979 Phase Transitions and Critical Phenomena vol 5b, ed C Domb and M S Green (New York: Academic)

[16] Poling D A and Parmenter R 1977 Phys. Rev. B 163240

[17] Poling D A 1982 J. Appl. Phys. 531864

[18] Stoller L 1987 Int. J. Theor. Phys. 26 755, 787, 805 